Copyright C1997, American Institute of Aeronautics and Astronautics, Inc.

AIAA Meeting Papers on Disc, January 1997

A9715215, AIAA Paper 97-0129

\title{
An experimental and computational study of bouncing and deformation in droplet collision
}

\author{
J. Qian \\ Princeton Univ., NJ \\ G. Tryggvason \\ Michigan Univ., Ann Arbor \\ C. K. Law \\ Princeton Univ., NJ
}

\begin{abstract}
AIAA, Aerospace Sciences Meeting \& Exhibit, 35th, Reno, NV, Jan. 6-9, 1997
An experimental and computational investigation of the collision of equal-sized liquid droplets was conducted. The Navier-Stokes equations for the fluid motion both inside and outside the droplets were solved using the numerical method of front tracking. The calculated history of the droplet collision process was found to agree well with the experimental results. The configuration of the gas gap between the droplet surfaces was analyzed in order to understand the mechanism which controls droplet bouncing. The minimum gap thickness was found to exhibit a nonmonotonic dependence on the droplet kinetic energy, thereby explaining the nonmonotonic transition between the collision regimes of coalescence and bouncing. Both computational and experimental results further showed that the droplet collision time is close to its natural oscillation period. Recognizing the large deformation of the droplet surface during collision, large amplitude droplet oscillation with viscous dissipation was also numerically studied. (Author)
\end{abstract}




\title{
An Experimental and Computational Study of Bouncing and Deformation in Droplet Collision
}

\author{
J. Qian ${ }^{1, *}$, G. Tryggvason ${ }^{2, * *}$, and C. K. Law ${ }^{1, * * *}$ \\ Department of Mechanical and Aerospace Engineering ${ }^{1}$ \\ Princeton University \\ Princeton, NJ 08544 \\ Department of Mechanical Engineering ${ }^{2}$ \\ The University of Michigan \\ Ann Arbor, MI 48109
}

\begin{abstract}
An experimental and computational investigation of the collision of equal-sized liquid droplets was conducted. The Navier-Stokes equations for the fluid motion both inside and outside the droplets were solved using the numerical method of "front tracking". The calculated history of the droplet collision process was found to agree well with the experimental results. The configuration of the gas gap between the droplet surfaces was analyzed in order to understand the mechanism which controls droplet bouncing. The minimum gap thickness was found to exhibit a non-monotonic dependence on the droplet kinetic energy, thereby explaining the non-monotonic transition between the collision regimes of coalescence and bouncing. Both computational and experimental results further showed that the droplet collision time is close to its natural oscillation period. Recognizing the large deformation of the droplet surface during collision, large amplitude droplet oscillation with viscous dissipation was also numerically studied.
\end{abstract}

\section{Introduction}

The dynamics of binary droplet collision is of interest to a variety of natural and technological problems including, for example, raindrop formation, nuclear fusion, as well as various spraying processes such as the spray painting, insecticide spraying, and spray combustion within liquidfueled combustors. A comprehensive understanding of the global phenomenology of droplet collision has emerged based on studies using water droplets (see, for example,

\section{* Graduate Student \\ ** Professor \\ *** Robert H. Goddard Professor, Fellow AIAA}

Copyright@1996 by C. K. Law. Published by the American Institute of Acronautics and Astronautics, Inc, with permission.
Adam \& Linblad 1968; Park1970; Brazier, Jennings \& Latham 1972; Ashgriz \& Poo 1990) and more recent studies using hydrocarbon droplets (Jiang, Umemura \& Law 1992; Qian \& Law 1996). Specifically, the collision outcome for a given liquid-gas system can be classified into five distinct regimes (figure 1) according to the collision Weber number (We) and collision impact parameter (B), which are respectively defined for identical droplets as $2 R p_{1} U^{2} / \sigma$ and $\chi /(2 R)$, with $R$ being the droplet radius, $U$ the relative velocity, $\chi$ the projection of the separation distance between the droplet centers in the direction normal to that of $U$, and $\rho_{1}$ and $\sigma$ the density and the surface tension of the liquid respectively. Thus $\mathrm{We}$ is the initial kinetic energy normalized by the droplet surface tension energy, while $B=0$ and 1 respectively designate head-on and grazing collisions.

The five regimes of collision outcome are respectively characterized by: (I) coalescence after minor droplet deformation, (II) bouncing, (III) coalescence after substantial droplet deformation, (IV) coalescence followed by separation for near head-on collisions, and (V) coalescence followed by separation for off-center collisions. Time-resolved photographic images of the collision sequence in these five regimes are shown in Jiang, Umemura \& Law (1992) and Qian \& Law (1996). A particularly interesting aspect of the collision outcome (Qian \& Law 1996) is the observed sensitive dependence of the boundary between bouncing and coalescence on the density of the gaseous medium. Increasing density by increasing either the pressure or the molecular weight of the gas medium promotes bouncing and widens Regime II, while the opposite holds for reduced density. In particular, by progressively reducing the environment pressure, Regime II first vanishes for $\mathrm{B}=0$ and eventually for all values of B. As such, while Regime II does not exist for the head-on collision of water droplets at one atmosphere pressure, it emerges as the system pressure is gradually increased to 2.7 atmospheres. It was suggested that the propensity for bouncing or merging is a consequence of the 
readiness with which the gaseous mass in the inter-droplet gap can be squeezed out of the gap by the colliding masses in order for the droplet surfaces to make contact.

Since in most instances the collision event involves large deformation, theoretical studies of the collision response have been mainly phenomenological in nature, for example through algebraic balances of force and energy. Advances, however, have been recently made on the numerical simulation of droplet collision. Specifically, adopting the front tracking method of Unverdi (1990) and Nobari \& Tryggvason (1994), which treats the multi-phase flow field as a single domain while delineating the different phases by tracking the interface movement, Nobari, Jan \& Tryggvason (1995) simulated the head-on collision event and gave a detailed description of the evolution of the droplet surface geometry as well as the pressure and velocity distribution in the entire flow field.

Recognizing the advantages offered by the numerical approach, especially those associated with large-scale deformation and the extremely small dimension of the inter-droplet spacing, which respectively are not readily amenable to analytical and experimental investigations, the present study was initiated with the overall objective of coupling the experimental and numerical results to gain further understanding of the droplet collision process. Three specific issues are addressed herein. First, we have perfonmed a quantitative comparison between the experimentally and computationally determined evolution of the droplet collision configurations. Since the experimental images can be determined quite precisely, they can be used as benchmark data for the validation of the computational code. Second, we have simulated the evolution of the inter-droplet configuration and related the results to the propensity of bouncing. Third, the droplet collision time has been simulated and experimentally determined, hence providing useful information on the characteristic time scale governing collision and its dependence on the impact inertia, surface tension, and viscous loss, for the situation of large deformation which is of particular interest here.

The numerical and experimental methods are specified in the next section, which is followed by presentation and discussion of the results.

\section{Formulation}

The front tracking numerical method adopted herein was developed by Unverdi (1990) and discussed in Unverdi \& Tryggvason (1992). The actual code is an axisymmetric version of the method, described in Jan \& Tryggvason (1995).

The physical problem and the computational domain is sketched in Fig. 2. The domain is axisymmetric, with the droplets initially placed near each end and the origin located at the mid-point of the line connecting the two droplet centers. The Navier-Stokes equations are valid for the fluid constituting the droplet as well as the ambient gas. A single set of momentum equations can be written for entire domain:

$$
\begin{aligned}
& \frac{2 R}{U T} \frac{\partial(\rho V)}{\partial t}+\Delta \cdot(\rho V V)=-\nabla p+g+ \\
& \nabla \cdot \frac{2 \mu}{\rho_{l} R U}\left(\nabla V+\nabla V^{T}\right)+\frac{8}{W e} \int \operatorname{mi} \delta\left(r-r_{f}\right) d a
\end{aligned}
$$

where $V, \rho$ and $p$ are the velocity, density and pressure, respectively normalized by half of the relative velocity $U / 2$, liquid density $\rho_{1}$ and the dynamic pressure $\rho_{1} U^{2} / 4$. Furthermore, $t$ is time normalized by the droplet oscillation time $T=2 \pi\left(\rho_{1} R^{3} / 8 \sigma\right)^{1 / 2}$, which is analytically derived based on inviscid droplet oscillation with small amplitude (Lamb 1932). In Eq. (1) surface tension is added as a delta function in the integration, resulting in a force distribution that is smooth and continuous over the droplet surface. Here $k$ is twice the mean curvature, $\mathbf{n}$ the unit normal vector of the droplet surface, $r$ the space vector, and the subscript $f$ designates the interface. Finally $g$ is a body force that is turned off at a prescribed droplet separation distance before collision, and is used to give the droplets an initial unity velocity toward each other.

We further note that $\rho$ is unity in the liquid phase but is given by $\gamma=\rho_{k} / \rho_{1}$ in the gas phase. Similarly, the effective viscosity coefficient, $2 \mu / \rho_{1} R U$, in equation (1) is $4 / R e$ and $4 \lambda / R e$ in the liquid and gas phases respectively, where $\lambda$ is the viscosity ratio between gas and liquid, and the Reynolds number is defined based on the liquid density and viscosity, as $2 p_{1} R U / \mu$. Therefore, the collision outcome depends on four nondimensional parameters: We, $\operatorname{Re}, \gamma$ and $\lambda$.

To solve the Navier-Stokes equation, we used a fixed, regular, staggered grid, a conservative, second order centered difference scheme for the spatial variables, and explicit first order time integration. In the computation mass is always conserved within a fraction of a percent. The interface is represented by separate computational points that are moved by interpolating their velocity from the grid. These points are connected to form a front which is used to keep the density and viscosity stratification sharp and to calculate the surface tension force.

The method and the code have been tested in various ways (Nobari 1993; Nobari, Jan \& Tryggvason 1995), for example by extensive grid refinement and comparison with published works (e.g. rising bubble, droplet oscillation). Figure 3 shows the droplet interface contour $h(r)$ from a test of grid refinement for water droplet collision at $t=0.63$, $W e=2.25$, and under $14.6 \mathrm{~atm}$ of argon. The deformed droplet surface shape and the gas gap configuration are 
compared for grids of 90 by 90 and 180 by 180 mesh points. It is seen that the two grids lead to essentially the same configuration, indicating that the code yields converged numerical result. Since the present numerical simulation is able to yield fine resolution of the geometry of the thin gap, which is of the order of $0.1 \mu \mathrm{m}$, all of the following numerical results were computed on a grid of 90 by 90 . The time required for each run ranged from 10 to 20 hours on a DEC 3000 workstation, depending on the governing parameters. Generally the method is very robust for moderate density ratios (about 100 or less), while at large density ratios difficulties in obtaining convergence in the pressure solver were sometimes encounted. In the present code we used a relatively simple SOR iteration to solve the pressure equation; other implementations of the method employ multigrid iteration which are generally more robust.

The experimental apparatus and procedure adopted are those of Jiang, Umemura \& Law (1992) and Qian \& Law (1996), in which two identical nozzle-generated streams of droplets of uniform size are made to impinge onto each other. Time-resolved images are obtained through stroboscopy synchronized with the droplet generation circuit. The entire apparatus is housed in a chamber whose pressure can be varied between 0.1 to 20 atmospheres. Furthermore, a digital imaging system accurately timeresolves the collision event, records the droplet image, and processes the data. This allows for fine resolution of the transition behavior between different regimes. The present investigation is concerned only with head-on collisions.

\section{Results}

In this section, we first show the numerical simulation of typical droplet collision sequences, and compare them with the experimental results. Then we consider the configuration of the inter-droplet gas gap during collision and relate it to the global response of coalescence and bouncing. Finally we study the droplet collision time, which will be shown to correspond mainly to the droplet oscillation time.

\section{Comparison of bouncing sequence}

Figures 4 and 5 compare the experimental (upper panel) and simulated (lower panel) images of the collision images for water and dodecanc droplets in 14.6 and 10 atmospheres of argon respectively. The simulations were conducted by using physical properties corresponding to those of the experiment. The collision in the experiment was slightly off-center, hence the existence of a small asymmetry in the axial direction. On the whole, however, the figures show that the computation quantitatively simulates well the experimental outcome of the collision. It is noteworthy to mention again that based on only the initial nondimensional numbers, $W e, \operatorname{Re}, \gamma$ and $\lambda$ measured at $t=0$ from experiments as the initial and boundary conditions, the simulation yielded all the subsequent detailed droplet surface geometry. Such a quantitative comparison on the droplet collision dynamics has not been conducted before. The close agreement substantiates the feasibility of the front tracking method in simulating droplet collision.

\section{Gas gap evolution}

When the droplets approach each other and gas is being squeezed out from the gap between the droplet surfaces, relatively high pressure is built up there, causing flattening of the droplets and conversion of the droplet kinetic energy into surface tension energy. The kinetic energy is also partly dissipated through the internal motion within the droplet and the gas flow. Onc limitation of the experimental observation is that the gap between the droplet surface can not be resolved because of its extremely small dimension. The success of the numerical simulation of droplet bouncing collision as shown above, and its ability to evaluate the gas gap configuration offers the opportunity for us to study the gap evolution in quantitative detail.

Figure 6(a) shows the half inter-droplet gap shape $h(r)$ during water droplet collision in 14.6 atmosphere argon with Weber number 2.25 at different time $t$ until the droplet reaches the maximum surface deformation. Note that as the two droplet surfaces approach each other, high pressure is built up in the direction of the lines of centers, causing indentations on the surfaces of the droplets in this region. It is seen that at $t=0.20$, the center region is slightly dented toward the liquid phase, and the minimum clearance takes the form of a ring concentric in the line of centers. It is also seen that the minimum clearance is right at the edge of the flattened surface. As the droplet surface approaches the maximum deformation with increasing flattening, the indentation in the center increases while the minimum clearance ring expands. After the droplets reach thcir maximum deformation at $t=0.63$, most of the initial droplet kinetic energy is transferred into surface tension energy. At this stage, the droplets start to contract because of the surface tension force, while the high gas pressure inside the gap pushes the droplets away. This is clearly seen in Fig. 6(b), which shows the gap evolution for $t \geq$ 0.63 after the droplets have reached the maximum deformation. As the droplets bounce away, the size of the minimum clearance ring gradually decreases while the gap thickness increases.

An interesting phenomenon observed from the gap evolution is the deformation of the droplet surface in the center region during collision. It is seen that the center 
indentation develops gradually until $t=0.63$. As the droplets bounce away, the indentation remains as shown in Fig. 6 (b). However, at $t=1.30$, the high pressure within the liquid pumps the indented surface out rapidly. This causes the minimum clearance position to switch from the ring to the center, and the minimum gap size first decreases and then increases again.

The development of the gas gap during tetradecane droplet collision at 14.6 atmosphere nitrogen with We $=7.42$ is shown in Figs. 7(a) and 7(b), which present similar characteristics observed during the gap evolution of water droplet collision. Specifically, the expanding and then shrinking of the minimum clearance ring during the collision and the "buckling" of the center indentation at the final collision stage are observed again.

Figure 8 plots the center clearance $h_{\text {center }}$ and minimum gap clearance at the rim $h_{r j m}$ as function of time $t$ for the above discussed water and tetradecane droplet collision. It shows that $h_{\text {center }}>h_{\text {rim }}$ during most of the collision time, as the minimum clearance ring stays away from the center. At the final collision stage, the center indentation buckles and the position of the minimum gap relocates to the center, causing $h_{\text {tim }}$ to equal $h_{\text {center }}$.

The study of gas gap evolution brings a critical question about the specific mechanism which controls the collision outcome of coalescence or bouncing. It is the gas pressure buildup which retards the droplet inter-surface movement and pushes the droplet away in the bouncing collision. Coalescence occurs when the gap size reaches the molecular interaction range, while bouncing occurs when the minimum gap is still larger than this range. Recognizing (Fig. 1) that as the droplet kinetic energy increases, the collision outcome changes from coalescence to bouncing, and then back to coalescence again, the variation of the gap size as the droplet kinetic energy changes has been calculated. Figure 9 plots the minimum gap width $h_{\min }$ as a function of the Weber number for decane, dodecane and tetradecane in 10 atmosphere nitrogen. Here $h_{\min }$ is the minimum value of $h_{\text {rim }}$, which represents the minimum distance between droplet surfaces during the entire collision process. It is seen that $\mathbf{h}_{\min }$ initially increases but then decreases as We increases. In other words, with small relative velocity the droplets can approach toward each other and coalesce readily, since the gas pressure buildup between the droplet surfaces is small. This is verified by the small deformation in regime I (Qian \& Law 1996). As the droplet kinetic energy increases, the gas pressure build up inside the gap is also increased which results in the large flattening of the surface and high gas resistance. The droplets can only approach each other until a minimum interface distance, which is larger than the molecular force range, and then bounce away (regime II). With further increase of the kinetic energy, however, the droplets can overcome the gas force and the minimum gap size is in the range of molecular force, resulting in coalescence again (regime III).

A series of experiments has been performed for decane, dodecane and tetradecane in 10 atmosphere nitrogen to evaluate the critical Weber numbers, $W e_{c, 1}$ and $W e_{c, 2}$ for the transitions between regime I and regime II, and between regime II and regime III respectively, as shown in Fig. 9. It is of interesting to note that, for a given fuel, the two critical Weber numbers yield values of $\mathbf{h}_{\mathbf{m} ! n}$ which lie on opposite sides of the maximum $\mathbf{h}_{\min }$, indicating a change of mechanism for bouncing and coalescence. Thus we have shown that the non-monotonic transition between the collision outcomes of coalescence and bouncing corresponds to a non-monotonic variation of the minimum gap size. It is also seen that the critical $h_{\min }$ 's are close, but not exactly equal. This is reasonable considering that the collisional dynamics including the extent of the viscous loss are quite different in these two regimes. It may be noted that in this study we have not addressed the issue of interfacial instability in effecting coalescence (Grumerman \& Homsy 1975, Hahn, Chen \& Slattery 1985, and Yiantsios \& Davis 1990). Clearly, if interfacial instability were important in causing rupturing of the thin gas film to effect coalescence, it becomes effective only when the film has reached some critical minimum distance which however is beyond the present photographic resolution of the image. Our results nevertheless do show that the film thickness is a primary controlling factor in determining whether the droplets coalesce or bounce away for relatively high kinetic energy collisions, and that this thickness varies in a nonmonotonic manner with the collision inertia.

It is also noted that the current investigation does not include molecular force in the numerical simulation. A fundamentally satisfactory description of droplet coalescence and bouncing must include the molecular attractive force which becomes operative as the dimension of the gap attain that of the molecular force range. It is anticipated that an analytical subgrid model for the flow in the film, with molecular force included, would result in more information of the gap evolution.

\section{Collision time}

An interesting observation from the present investigation is that the droplet collision time is close to the natural oscillation time $\mathrm{T}$ of the droplet. Such a result has been observed numerically by, for example Foote (1975) and recently Nobari, Jan and Tryggvason (1995). A direct comparison between experimental and computed results, however, has not been conducted. We have therefore 
performed a series of experiments and the corresponding numerical simulation for the collision time of water and tetradecane droplets with different Weber numbers in 14.6 atmosphere argon. The experimental collision time, say $\tau$, is measured as the time when droplets are visually in contact. Figure 4(a) shows that the time interval between the state when the droplet surfaces first come to contact at $t=0.12$, and the state when the droplets start to bounce away at $t=1.26$ is 1.14 . Figure 5 (a) shows a collision time of about 0.98 . The numerical collision time is defined as the period between the state when the slope of the gap center clearance $h_{\text {center }}$ starts to level off until the state at which $h_{\text {center }}$ starts to increase. Figure 10 shows that these two states are sharply defined in terms of $h_{\text {center }}$, implying that this collision time is well defined. Figures 11 and 12 show that the experimentally and numerically determined collision times indeed agree well.

Figure 13 plots $h_{m}, c$, which is the calculated half distance between the mass centers of the droplets, as function of time $t$ when the droplets collide and bounce away, with $\mathrm{We}=6.96$. The variation of $h_{\mathrm{m}, \mathrm{c}}$ can be divided into two stages corresponding to two time intervals, say $\tau_{1}$ and $\tau_{2}$, as shown in Fig. 13. The first stage is between the states when the droplet surface starts to deform at $h_{m, c}=1$ until the state when it has achieved maximum deformation with $h_{m, c}$ attaining a minimum value. The second stage is between the maximum deformation state and when the droplets become basically non-interactive again at $h_{m, c}=1$. Note that the first stage is mainly affected by the droplet impact inertia as the droplet surface deforms upon impact, and $\tau_{1}$ decreases as the droplet kinetic energy increases, which is shown in Figs. 14 (a) and 14(b) for water and tetradecane in 14.6 atmosphere argon. The second stage is dynamically similar to droplet oscillation, starting from the maximum deformed shape, and decreases gradually as We increases, as shown in Figs. 14(a) and 14(b). The sum of $\tau_{1}$ and $\tau_{2}, \tau_{\mathrm{m}, \mathrm{c}}=\tau_{1}+\tau_{2}$, is another way to describe the collision time as shown in Figs. 11 and 12. Here $\tau$ is larger than $\tau_{\mathrm{m}, \mathrm{c}}$ because of the fact that the droplets are deformed into an elongated shape in the direction of motion (see Figs. 4 and 5) when they bounce away such that $h_{m, c}$ is larger than one. For a rough estimate of the collision time, we approximate $\tau_{1}$ as $(2 \mathrm{R} / \mathrm{U}) / \mathrm{T}=1 / \mathrm{We}^{1 / 2}$ and $\tau_{2}$ as half of the collision time, 0.5 , so the collision time is around $1 / \mathrm{We}^{1 / 2}+0.5$. Since the Weber numbers in the bouncing collision regime are mostly in the range of 1 to 10 , the collision time is therefore close to unity. This explains the numerical and experimental observation that the collision time in droplet bouncing is close to the droplet oscillation time and decreases with initial kinetic energy. To further explore the close relationship between the droplet collision time and the oscillation time, we study the oscillation of an isolated, initially oblate spheroidal droplet, whose cross sectional area is an ellipse with two principal radii $\alpha$ and $\beta$ nondimensionalized by $R$. Figures $15(\mathrm{a})$ and 15 (b) respectively show the images of the oscillation of a tetradecane droplet with initial deformations of $\alpha_{0}=1.1$ and 1.4. Figure 16 plots the oscillation of $\alpha$ with $t$, and demonstrates that the extra surface tension energy is quickly dissipated by viscous action such that $\alpha$ approaches unity in a couple of cycles. It is also seen that the first cycle time $T^{*}$, defined as the time lapse between the first two largest deformations, is 1.40 for $\alpha_{0}=1.1$ and 1.2 for $\alpha_{0}=1.4$. Thus the oscillation time decreases with increasing initial deformation.

To further consider the effects of viscous action and finite deformation on the droplet oscillation cycle time, we have evaluated the oscillation period $T^{*}$ as a function of the Ohnesorge number, $Z=\mu_{1} /(\rho R \sigma)$, which represents the viscous effect. Figure 17 plots $T^{+}$as function of $\alpha_{0}$ for different $\mathrm{Z}$, where $\mathrm{Z}=0.088$ is representative of the rheological properties of tetradecane. It is seen that $T^{*}$ is larger for large values of $\mathrm{Z}$, indicating that viscous dissipation results in longer cycle times. It is again observed that $T^{*}$ decreases as $\alpha_{0}$ increases, implying that large initial deformation leads to shorter cycle time.

\section{Conclusions}

The present investigation presents a detailed experimental and computational study of droplet bouncing collision. The computational method of "front tracking" was able to accurately simulate the droplet collision process, and the numerical predictions agree well with the experimental results. The evolution of the gas gap between the droplet surfaces during the collision was computed, and it was observed that the minimum clearance of the gap assumes the shape of a ring concentric with the line of centers, and the indentation in the center region remains during most of the collision event until the droplets have sufficiently bounced away, at which the high liquid pressure rapidly buckles the indented surface. The minimum gap thickness during collision shows a non-monotonic dependence on the kinetic energy, which is shown to be related to the nonmonotonic response of the collision outcome, which can change from coalescence to bouncing, and then back to coalescence as the collision Weber number increases. Finally, we demonstrated that the collision time is mainly controlled by the droplet oscillation time, which is in turn affected by droplet viscous dissipation and surface deformation.

\section{Acknowledgment}

The research at Princeton and the University of Michigan were respectively supported by the Air Force Office of Scientific Research and NASA. Computatioanl time was provided by the NSF Pittsburgh Supercomputing Center. 


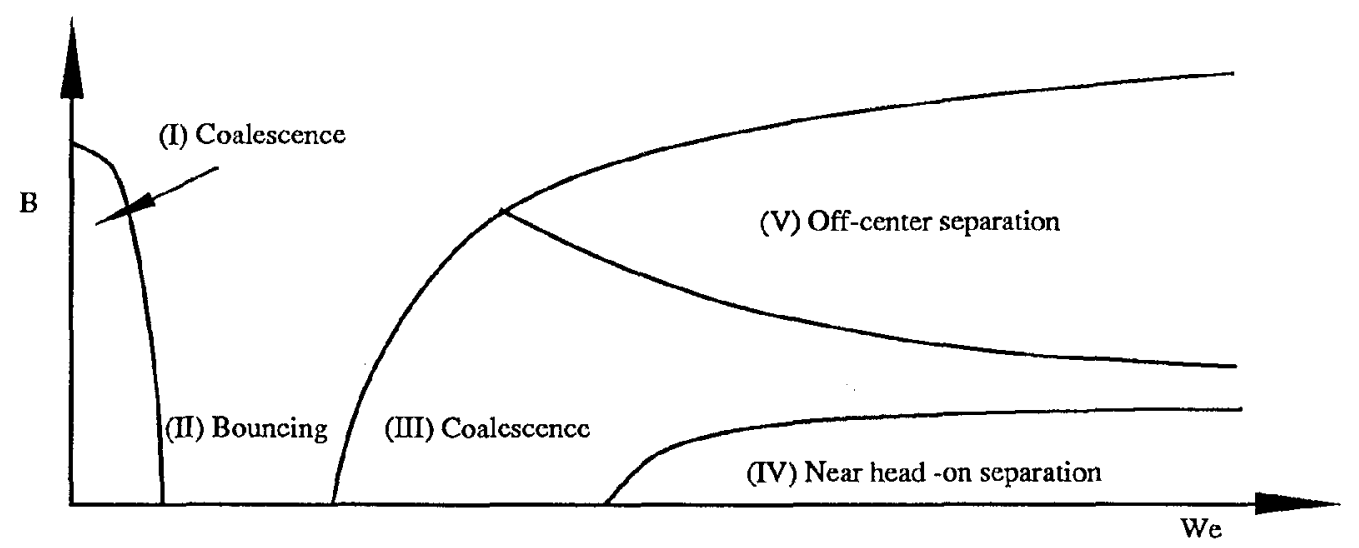

Figure 1. Schematic of different droplet collision regimes as function of Weber number (We) and impact number (B).

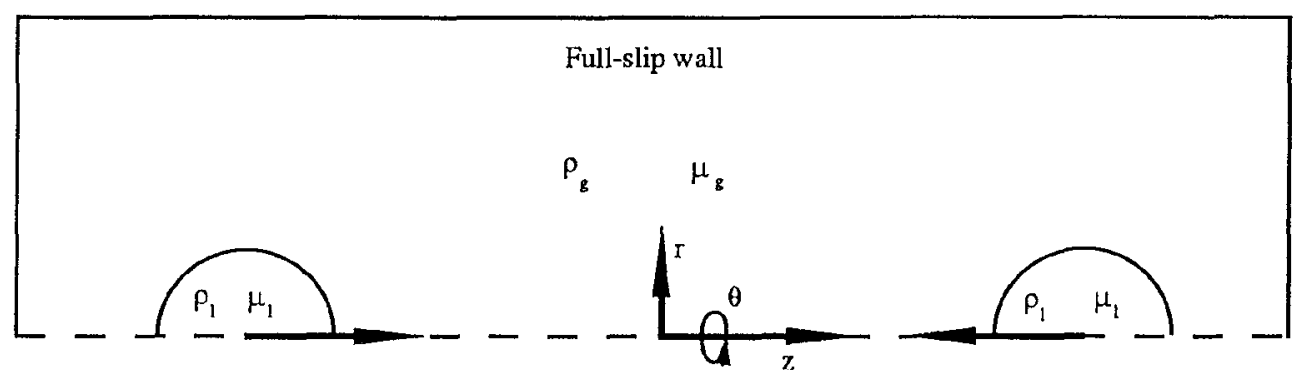

Figure 2. The computational setup of axisymmetric domain, which is bounded by full-slip walls and resolved by a regular grid.
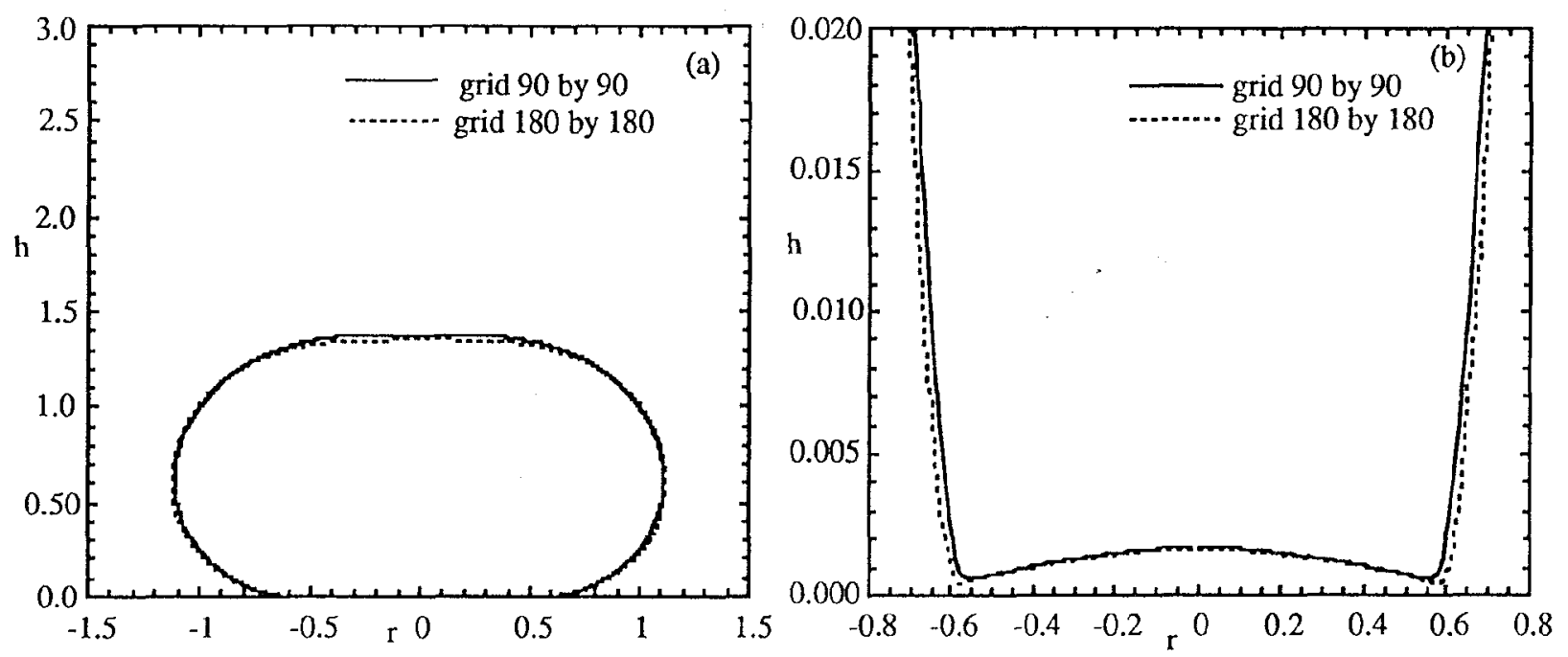

Figure 3. Resolution test. Droplet shape and gas gap configuration from computation with 90 by 90 grid are compared with results obtained with 180 by 180 grid; (a) entire droplet, (b) inte-droplet spacing. 

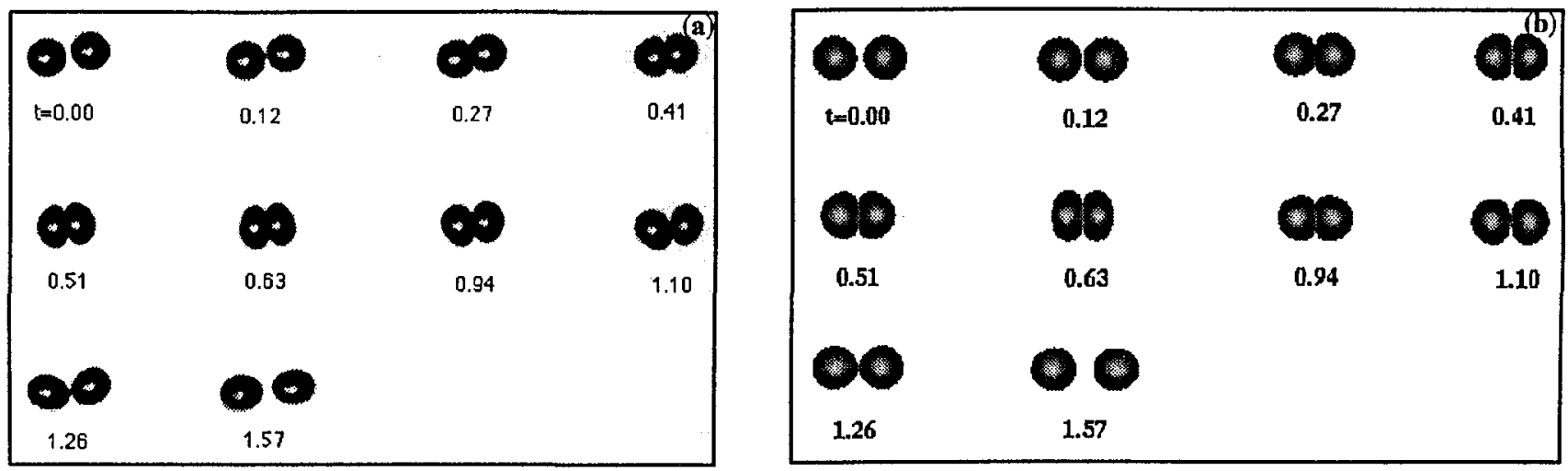

Figure 4. (a) Experimental recordings and (b) numerical simulation of water droplet head-on collision sequence in $14.6 \mathrm{~atm}$. argon with We=7.42.
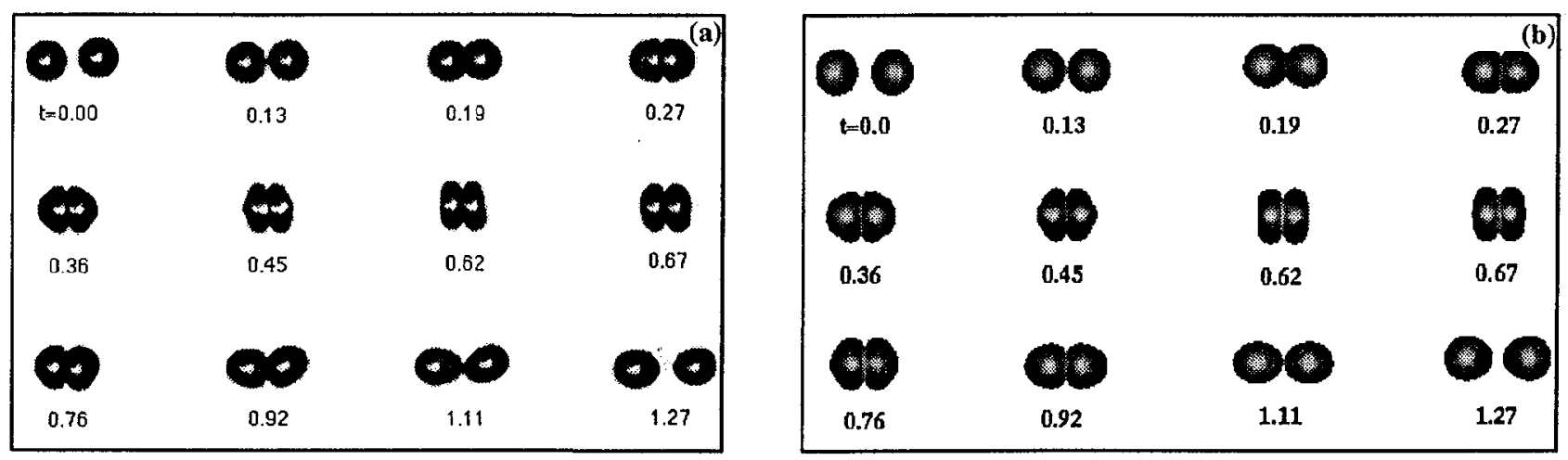

Figure 5. (a) Experimental recordings and (b) numerical simulation of tetradecane droplet head-on collision in $10 \mathrm{~atm}$. nitrogen with $\mathrm{We}=\mathbf{7 . 4 2}$. 

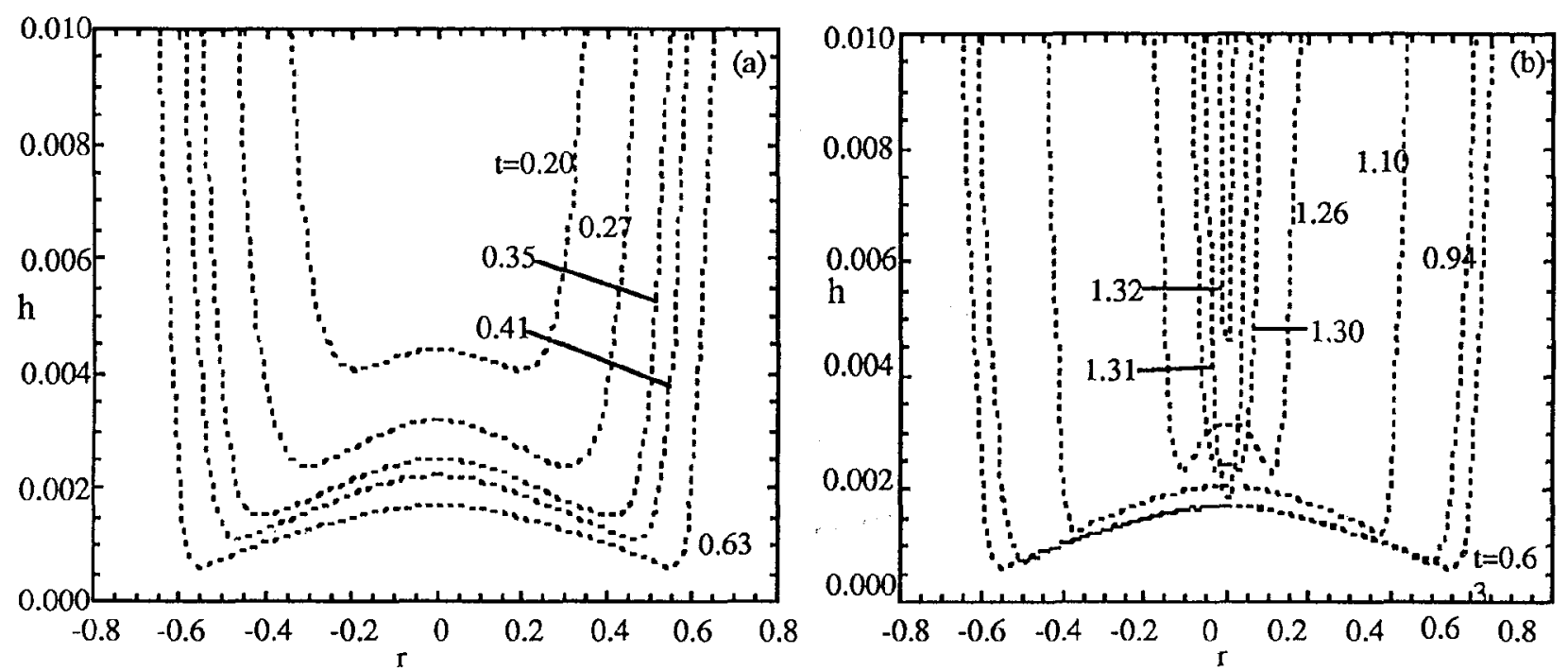

Figure 6. Inter-droplet gap geometry evolution during water droplet collision in 14.6 atm. argon with $\mathrm{We}=2.25$; (a) approaching sequence, (b) receding sequence.
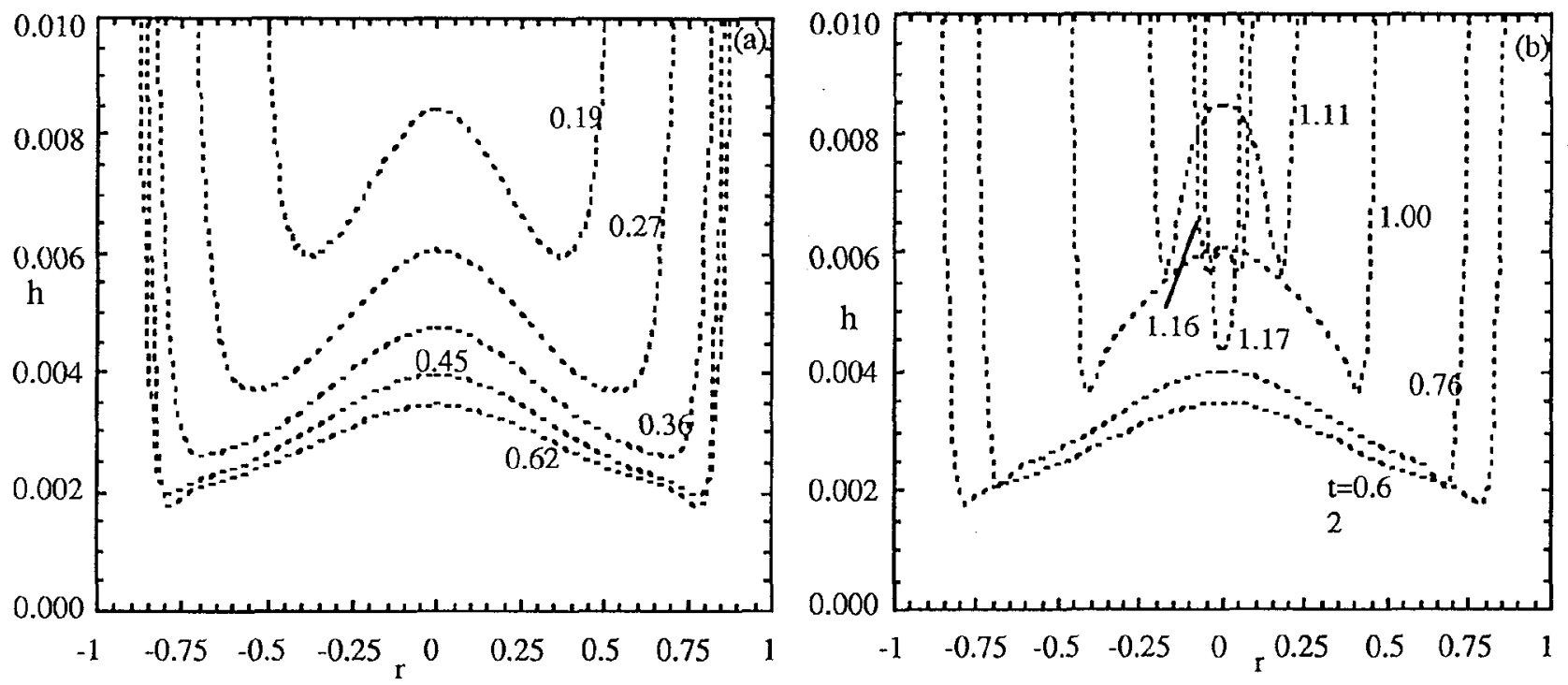

Figure 7. Inter-droplet gap geometry evolution during tetradecane droplet collision in $10 \mathrm{~atm}$. nitrogen with $\mathrm{We}=7.42$; (a) approaching sequence, (b) receding sequence. 


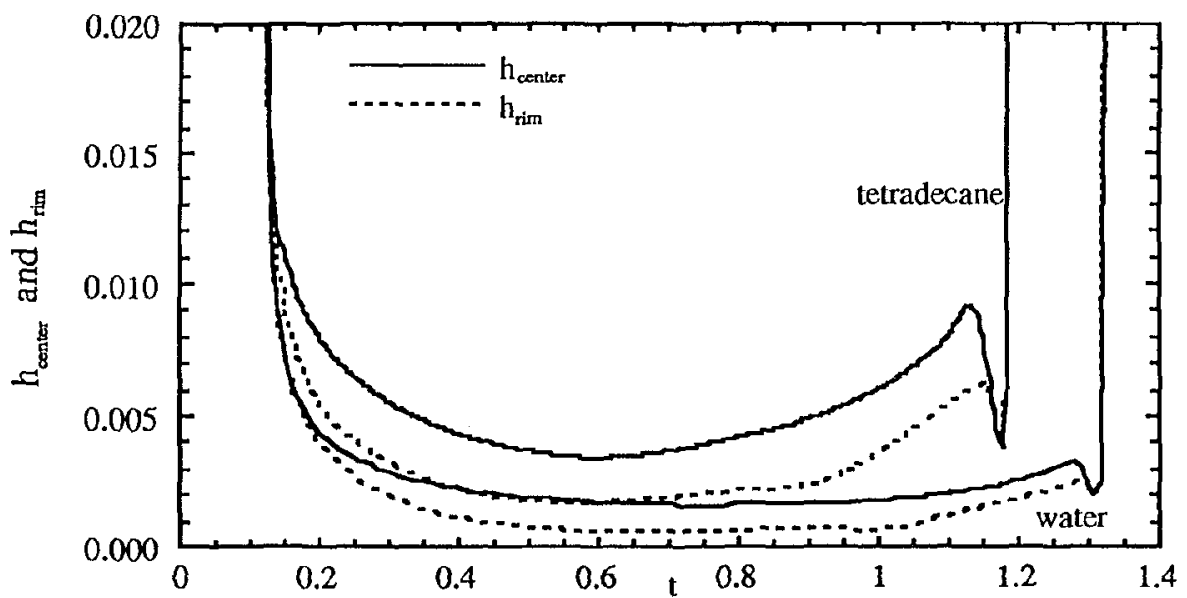

Figure 8. The evolution of $h_{\text {conter }}$ and $h_{\text {rim }}$ for the water and tetradecane droplet collision sequence of Figs. 6 and 7 .

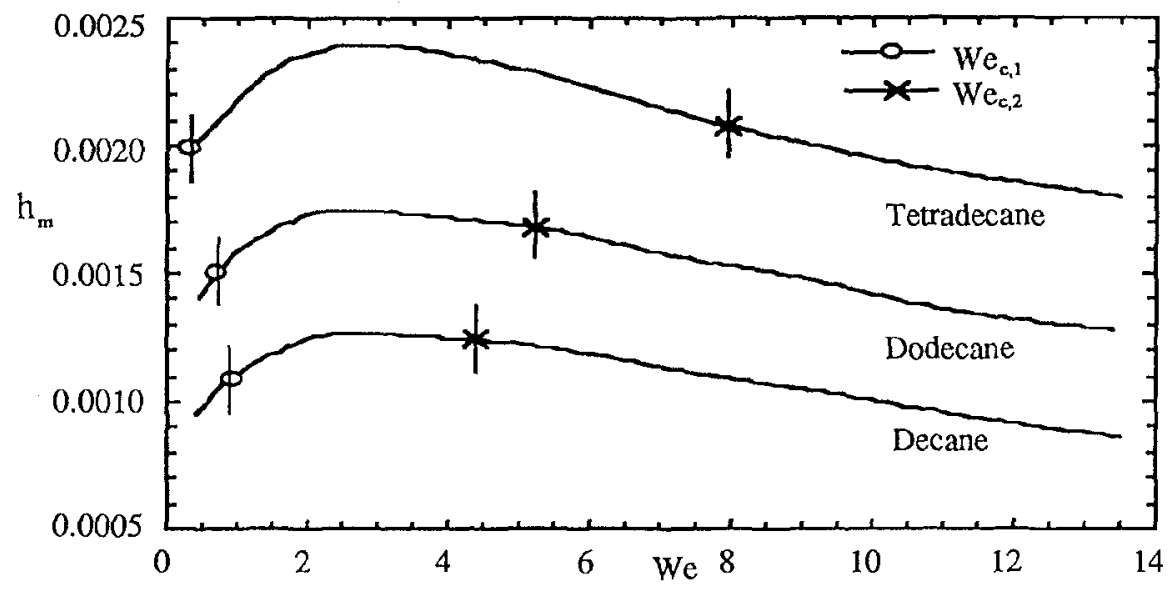

Figure 9. Minimum gap distance during the entire collision sequence as function of Weber number for decane, dodecane and tetradecanc.

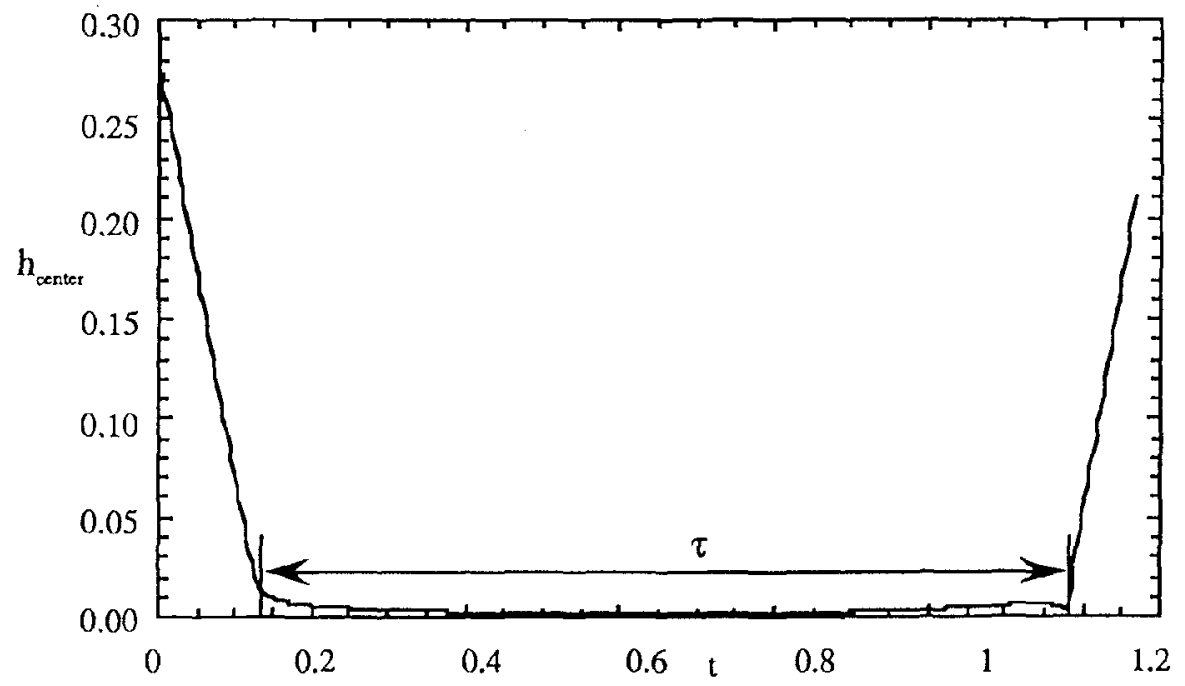

Figure 10. Calculated inter-center distance as function of time, which defines the collision time. 


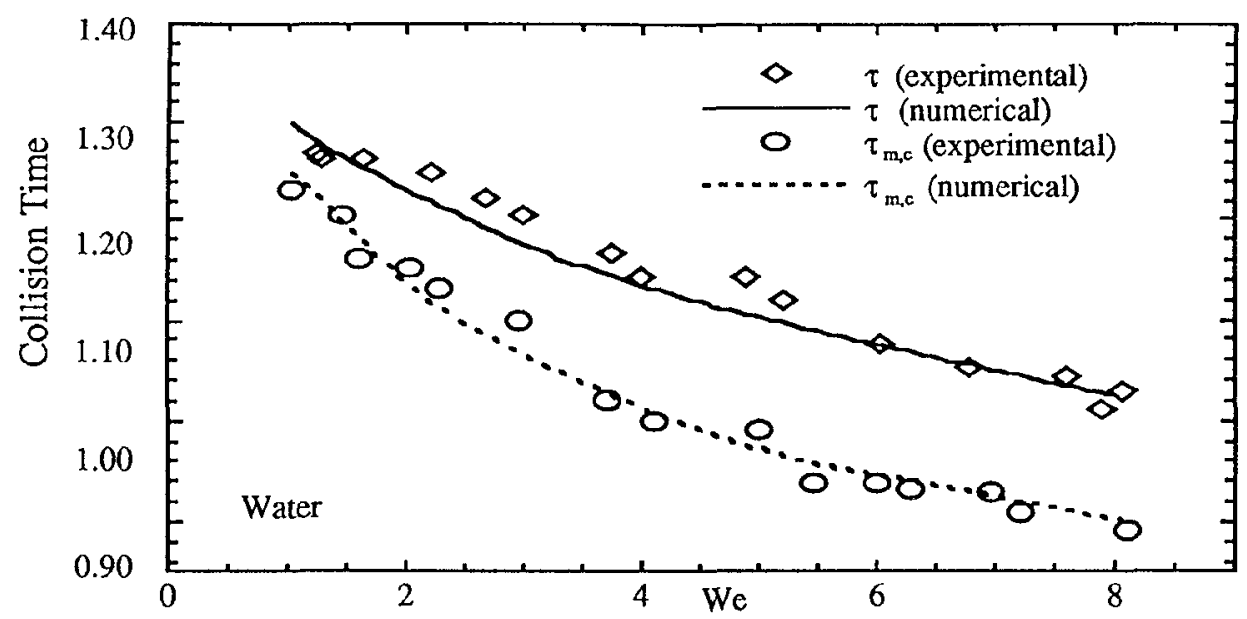

Figure 11. Collision time as function of Weber number for water droplets.

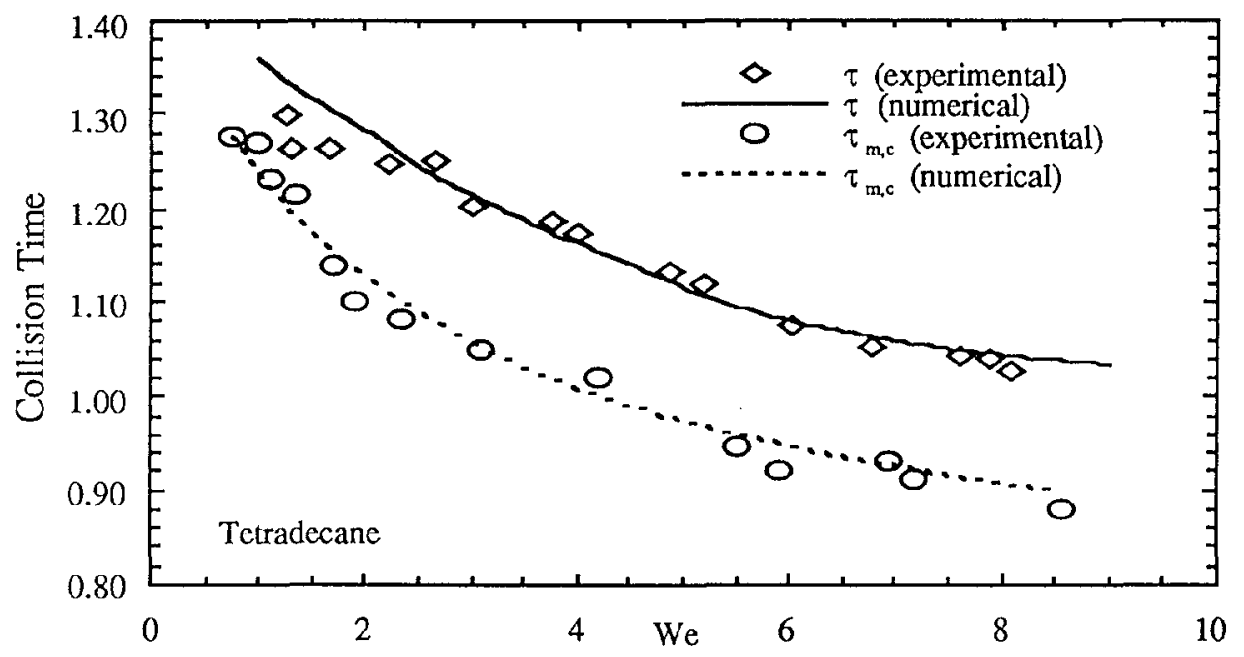

Figure 12. Collision time as function of Weber number for tetradecane droplets.

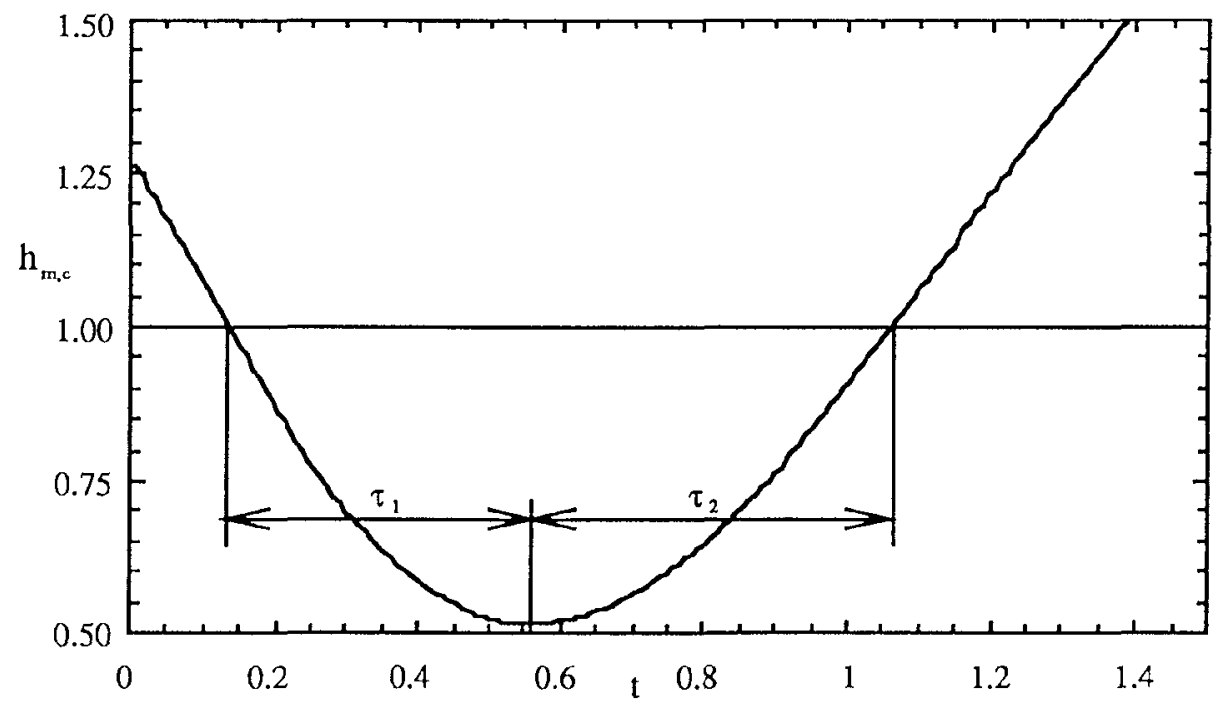

Figure 13. The variation of mass center of droplet with time during tetradecane droplet collision with $\mathrm{We}=6.96$ in $14.6 \mathrm{~atm}$. argon. 
Copyright (C)1997, American Institute of Aeronautics and Astronautics, Inc.
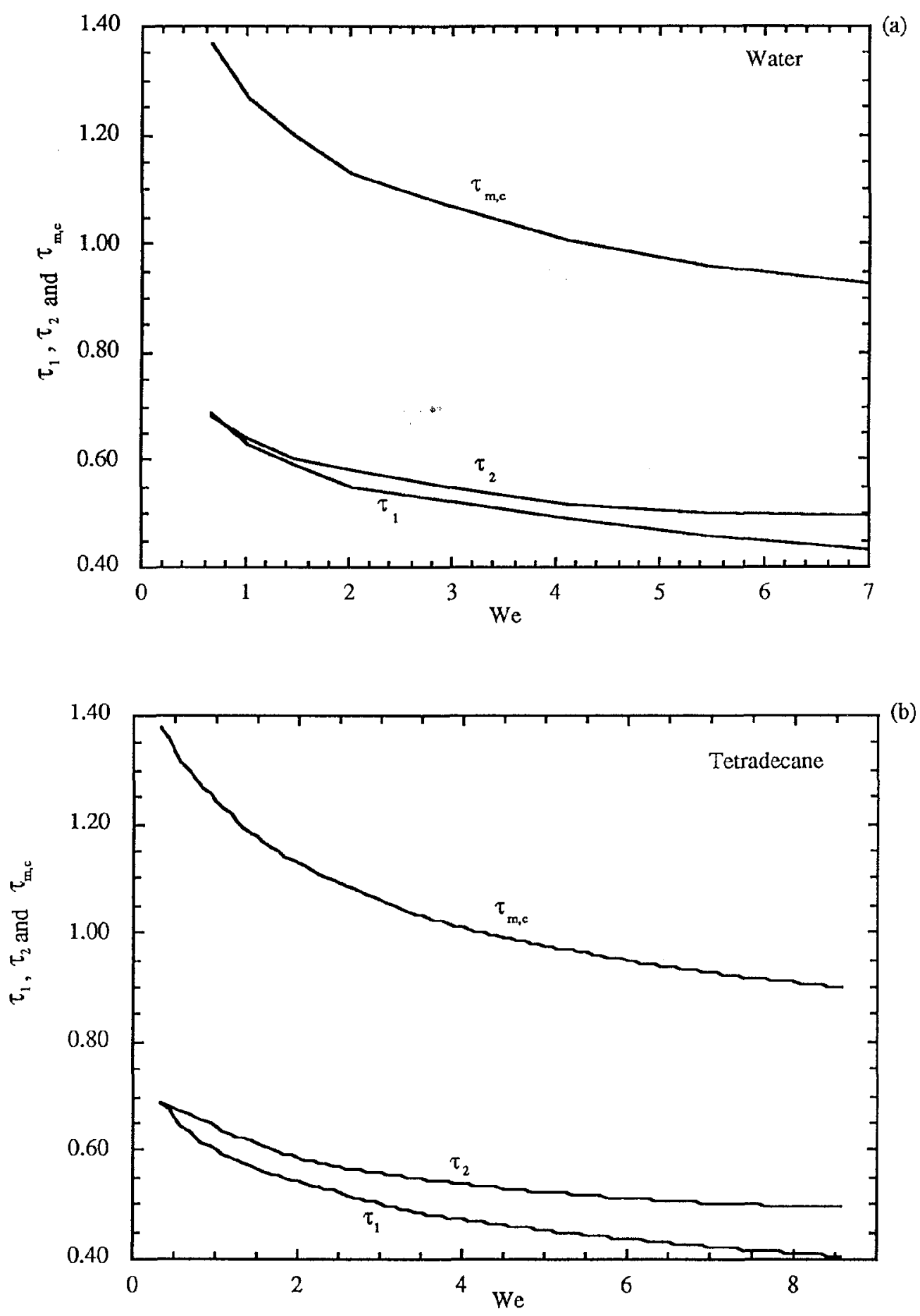

Figure 14. Collision time as function of We for (a) water and (b) tetradecane in $14.6 \mathrm{~atm}$. argon. 
Copyright (C)1997, American Institute of Aeronautics and Astronautics, Inc.

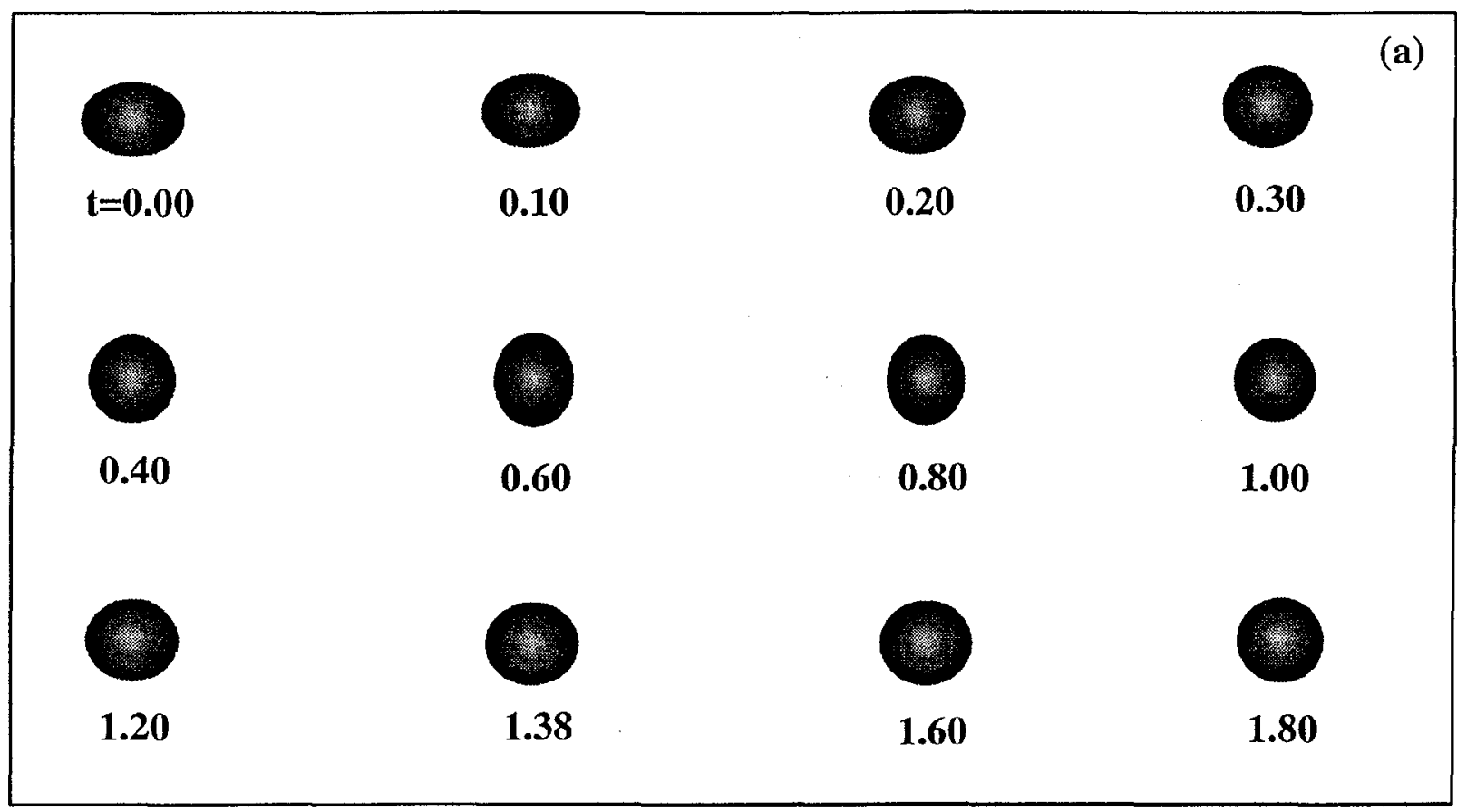

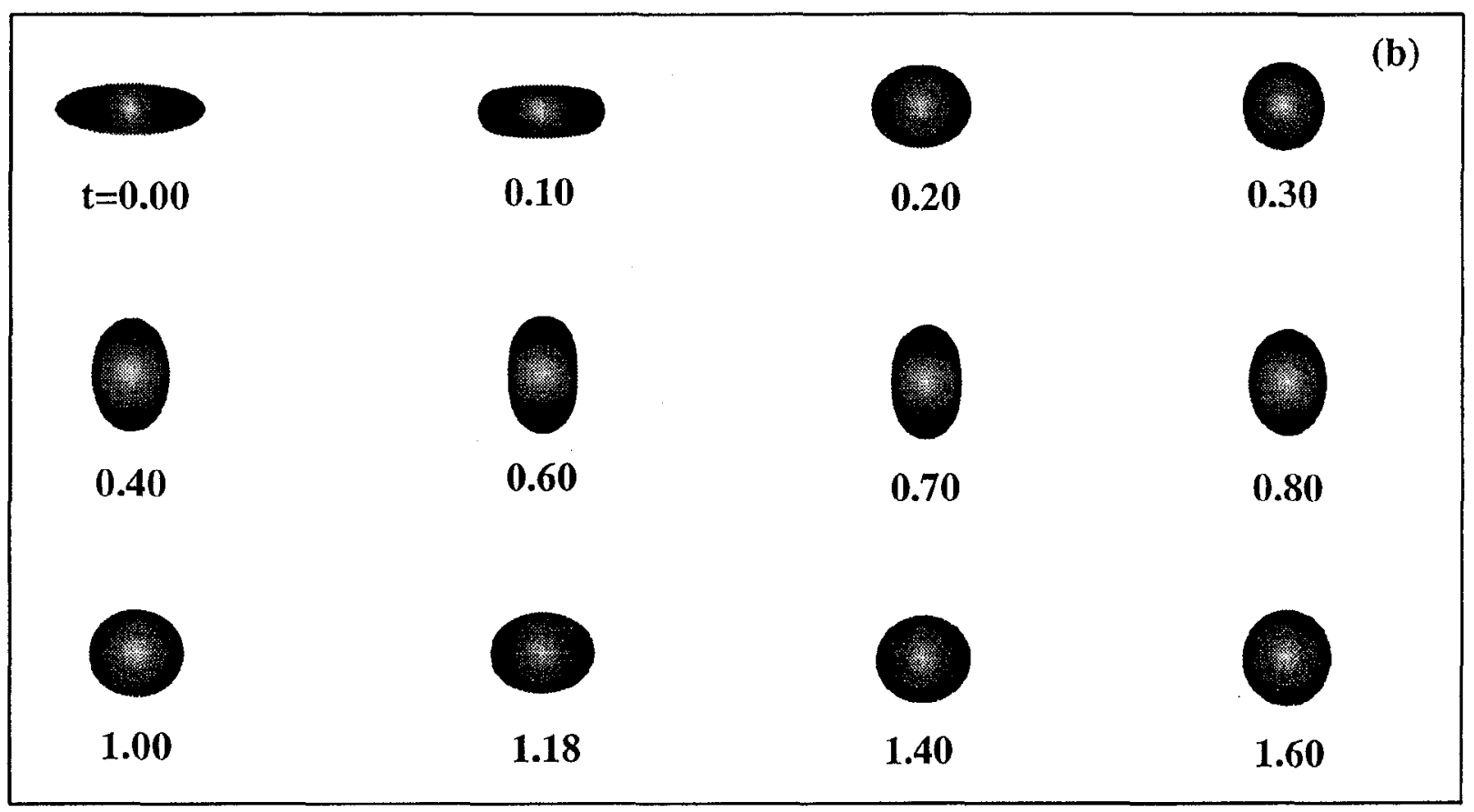

Figure 15. Numerical simulation of droplet oscillation with different initial deformation. 


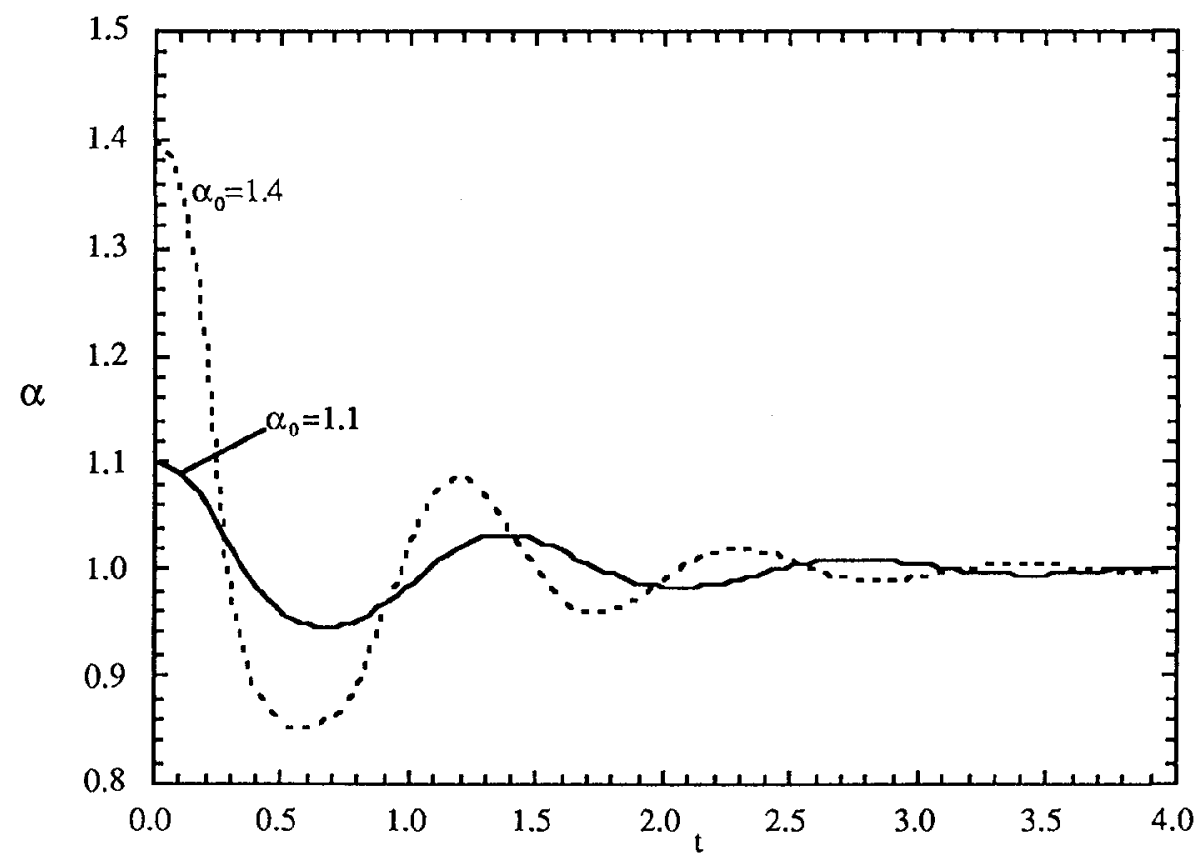

Figure 16. Oscillation of a tetradecane droplet with different initial deformation.

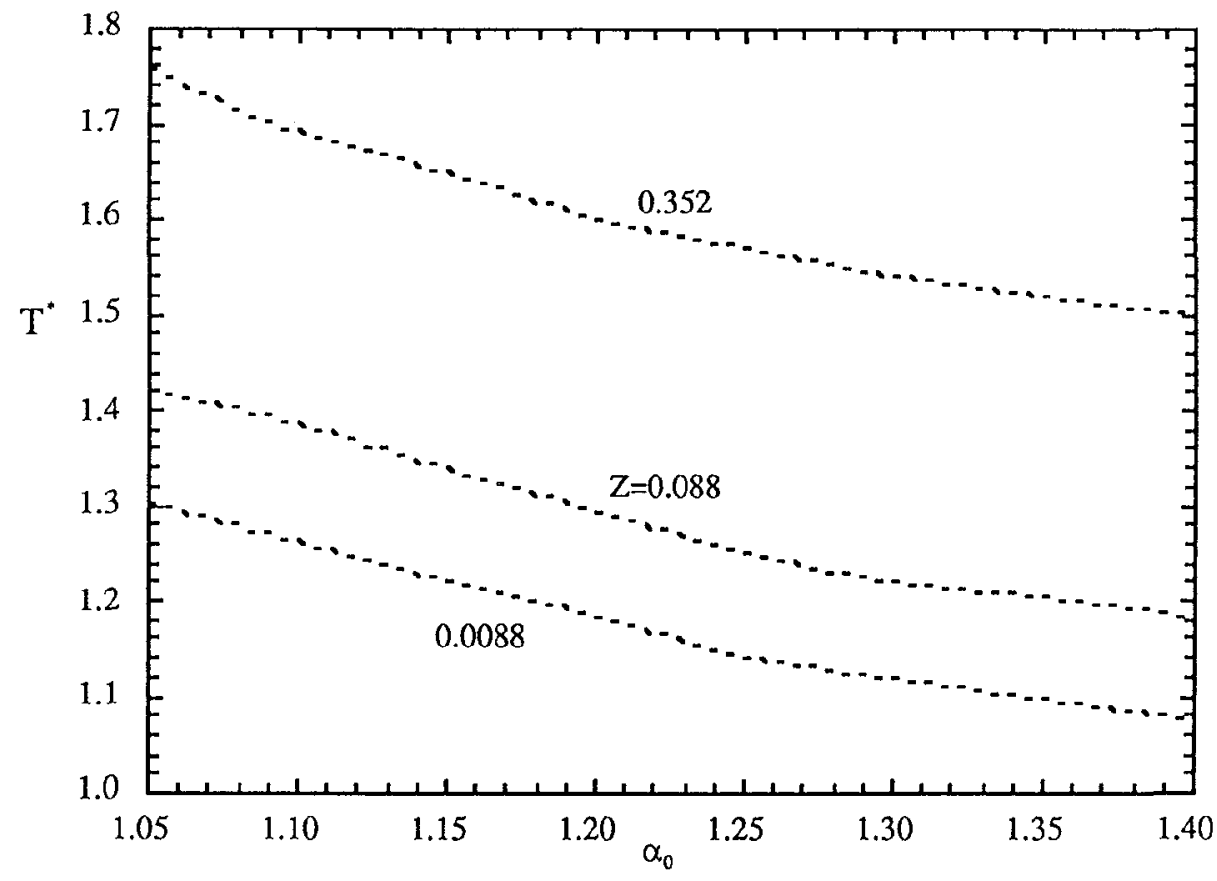

Figure 17. First oscillation period as function of initial deformation for different Ohnesorge number. 